\title{
Factores de riesgo asociados con infección de la vía urinaria provocada por superbacterias
}

\section{Risk factors associated with developing urinary tract infections caused by superbugs}

\author{
Alan Johan Chavolla-Canal, ${ }^{1}$ Mirna Gisel González-Mercado²
}

\begin{abstract}
Resumen
ANTECEDENTES: En los últimos años ha tomado relevancia la aparición de resistencia panfarmacológica de superbacterias en pacientes con infección de la vía urinaria. Aunque se han descrito algunos factores de riesgo, son pocas las publicaciones al respecto. OBJETIVO: Analizar en la población mexicana los factores de riesgo relacionados con la infección de la vía urinaria provocada por superbacterias.

MATERIALES Y MÉTODOS: Estudio descriptivo y retrospectivo, en el que se analizaron los expedientes de urocultivos efectuados entre enero de 2007 y junio de 2015. Las variables de estudio fueron: sexo, edad, lugar de tratamiento, enfermedades relacionadas, antecedente de cirugías, servicio a cargo, razón de hospitalización, transfusión, tratamiento, sonda urinaria, cultivo de control y defunción. Para el análisis de los datos se utilizó estadística descriptiva.

RESULTADOS: Se registraron 31 pacientes con infección urinaria provocada por superbacterias. Pseudomonas aeruginosa fue el microorganismo aislado con mayor frecuencia, predominantemente en hombres y en pacientes de la tercera edad. Las enfermedades con mayor prevalencia fueron: diabetes e hipertensión; el antecedente de transfusión y colocación de sonda urinaria representó un factor de riesgo asociado con la infección y tasa de mortalidad. Los antibióticos más prescritos fueron las cefalosporinas y la mortalidad general fue de $38.7 \%$.

CONCLUSIÓN: Ante la repercusión mundial de la resistencia panfarmacológica a los antibióticos hacen falta más estudios que contribuyan a disminuir la tasa de mortalidad. Este estudio proporciona información valiosa de los factores de riesgo asociados con infección de la vía urinaria por superbacterias.
\end{abstract}

PALABRAS CLAVE: Factores de riesgo; cultivo de control urinario; superbacterias; Pseudomonas aeruginosa.

Abstract

BACKGROUND: The recent appearance of pandrug resistance due to superbugs has become relevant in patients with urinary tract infections. Some risk factors have been described, but there are few studies published on the topic.

AIM: To analyze the risk factors related to urinary tract infections due to superbugs in the Mexican population.

MATERIALS AND METHODS: In a descriptive, retrospective study, the medical records of patients that had urine cultures within the time frame of January 2007 to June 2015 were analyzed. The study variables were: sex, age, place of treatment, related diseases, prior surgeries, service in charge of the patient, reason for hospitalization, transfusion, treatment, indwelling urinary catheter, control culture, and death. Descriptive statistics were used for the data analysis.

\footnotetext{
${ }^{1}$ Servicio de Urología, Hospital General Regional 46 (IMSS) de Guadalajara, Jalisco; jefe de Cirugía del Hospital San Javier, Nuevo Vallarta, Nayarit.

2 División de Biotecnología y Salud, Tecnológico de Monterrey, Guadalajara, Jalisco.
}

Recibido: marzo 2018

Aceptado: octubre 2018

Correspondencia

Alan Johan Chavolla Canal

radiohead_56@hotmail.com

Este artículo debe citarse como

Chavolla-Canal AJ, González-Mercado MG. Factores de riesgo asociados con infección de la vía urinaria provocada por superbacterias. Rev Mex Urol. 2018 nov-dic;78(6):425-33.

DOI: https://doi.org/10.24245/revmexurol.v78i6.2111 
RESULTS: Thirty-one patients with superbug urinary infections were registered in the study. The most frequently isolated microorganism was Pseudomonas aeruginosa, predominating in men and advanced-age patients. Diabetes and high blood pressure were the most prevalent diseases. A history of transfusions and urinary catheter placement were risk factors associated with infection and mortality. Cephalosporins were the most widely prescribed antibiotics and the general mortality rate was $38.7 \%$.

CONCLUSION: More research is needed on this topic, given the worldwide impact of infections that are resistant to the majority of drugs on antibiotic panels. The present study provided valuable information on the risk factors associated with the devastating phenomenon of pandrug resistant infection caused by superbugs.

KEYWORDS: Risk factors; Control urine culture; Superbugs; Pseudomonas aeruginosa.

\section{ANTECEDENTES}

La infección de la vía urinaria es una enfermedad provocada por la invasión, colonización y multiplicación de microorganismos. ${ }^{1}$ La elevada prevalencia de la infección repercute de manera negativa en la tasa de consultas, hospitalización, gastos en salud, además de ser responsable de aproximadamente $38 \%$ de las infecciones nosocomiales, y de éstas $80 \%$ relacionadas con la colocación de catéter transuretral. Escherichia coli se encuentra en $85 \%$ de los cultivos extrahospitalarios, 50\% intrahospitalarios y $95 \%$ de las infecciones monobacterianas. ${ }^{1,2}$ Los agentes etiológicos más frecuentemente identificados en pacientes con infección de vías urinarias incluyen: E. coli, Klebsiella sp, estafilococos, enterobacterias, Proteus mirabilis, Pseudomonas aeruginosa y especies de enterococos. ${ }^{3}$

La resistencia bacteriana es un fenómeno caracterizado por la facultad de adaptación de los microorganismos a las agresiones de su medio, incluso antes del descubrimiento de los antibióticos; sin embargo, con su advenimiento se ha intensificado por el proceso de presión selectiva. La resistencia bacteriana disminuye la posibilidad de curación clínica y erradicación del agente causal, además de incrementar los costos de tratamiento, la morbilidad y mortalidad. ${ }^{4}$
Diversos estudios reportan el aumento de bacterias multirresistentes causantes de infecciones de la vía urinaria; ${ }^{5-6}$ por ejemplo, Pseudomonas aeruginosa es uno de los microorganismos aislados con mayor capacidad multirresistente y hace poco se le identificó un fenómeno facultativo de resistencia bacteriana extensa y de resistencia panfarmacológica. ${ }^{7}$

La multirresistencia se define como la ausencia de sensibilidad a un fármaco, como mínimo, en tres o más de las categorías de antibióticos. Cuando la ausencia de sensibilidad ocurre en la mayor parte de los antimicrobianos, excepto en dos categorías, se denomina resistencia extensa, mientras que la resistencia a todos los antimicrobianos se define como panfarmacológica, que en este caso se utiliza el término de superbacteria al agente causal de la infección..$^{4-7}$ En este aspecto las bacterias más frecuentemente identificadas en pacientes con infecciones de vías urinarias son Pseudomonas aeruginosa y Acinetobacter baumannii. ${ }^{4-8}$

Existen dos factores que favorecen el aumento de infecciones provocadas por estos microorganismos: 1) la prescripción indiscriminada de antimicrobianos y 2) la rápida evolución de mecanismos de resistencia entre las bacterias. Pseudomonas aeruginosa suele afectar a pacientes inmunodeprimidos, con quemaduras 
de tercer grado, fibrosis quística, y quienes permanecen en la unidad de cuidados intensivos, entre otros.

En América Latina se registran los porcentajes más altos de resistencia antimicrobiana, comparados con algunas regiones de Estados Unidos y Europa, que reportan tasas de resistencia a carbapenémicos de $25 \%$, excepto en Grecia, con $51 \%$. Diversos estudios señalan que Pseudomonas aeruginosa representa el cuarto microorganismo multirresistente aislado de la unidad de terapia intensiva, seguido de E. coli, S. aureus y Klebsiella pneumoniae. ${ }^{8}$

Los mecanismos implicados en la resistencia de este tipo de superbacterias incluyen: reducción de la permeabilidad de la membrana plasmática, bombas de expulsión de los fármacos, beta-lactamasa cromosómica y plasmídica, carbapenemasa, acetil-transferasa, entre otros. De esta forma, solo permanece la opción de indicar medicamentos con alto grado de toxicidad, como las polimixinas. ${ }^{6,8}$

A partir del año 2001 la Organización Mundial de la Salud consideró como urgencia y prioridad este problema de salud, por lo que instituyó las medidas globales para la contención de la resistencia antimicrobiana, que incluye reportes epidemiológicos de todas las regiones con periodicidad e informe de los factores de riesgo asociados. De ahí la importancia de publicar este fenómeno en todo el mundo, con especial atención en los factores de riesgo para lograr dicha contención. ${ }^{9}$

Los factores de riesgo de infección de vías urinarias reportados son: disminución del consumo de líquidos, diferir la micción, actividad sexual, manipulación y calidad de la flora vaginal. En mujeres: la menor longitud de la uretra y su terminación en el introito facilita su colonización; malformaciones congénitas (especialmente en niños), hiperplasia prostática, cálculos o tumores, vaciamiento vesical incompleto, reflujo vesicoureteral, etc. ${ }^{10,11}$

Los factores de riesgo identificados para infecciones con multirresistencia y resistencia extensa son: indicación de antibióticos previamente (particularmente aminoglucósidos), antecedente de cirugías, colocación de catéter urinario, admisiones hospitalarias previas y, como enfermedades relacionadas, alteraciones metabólicas, inmunodeficiencias y cáncer. ${ }^{6,8-14}$

La resistencia bacteriana es un fenómeno ampliamente conocido y descrito en la bibliografía, y su relación con la prescripción de antimicrobianos; sin embargo, México no cuenta con suficientes estudios al respecto. ${ }^{4}$

La mayor parte de las investigaciones describe factores de riesgo asociados con bacterias multirresistentes, otras se relacionan con resistencia extensa y muy pocas reportan factores de riesgo asociados con superbacterias. Sin embargo, no existe evidencia de reportes de factores de riesgo para infecciones por superbacterias aisladas de la vía urinaria.

Con base en lo anterior, el objetivo de este estudio fue: evaluar la evidencia de factores de riesgo asociados con infección de vías urinarias provocada por superbacterias en la población mexicana.

\section{MATERIALES Y MÉTODOS}

Estudio descriptivo y retrospectivo, con registro de aprobación del comité interno hospitalario R-2017-1303-112, efectuado mediante la revisión y análisis de antibiogramas de urocultivos del Laboratorio de Bacteriología del Hospital General Regional 46 del estado de Jalisco, entre enero de 2007 y junio de 2015. Para el desarrollo del estudio se tomó en cuenta el conteo mayor 
de $10^{5}$ unidades formadoras de colonias (UFC) como indicativo de bacteriuria significativa y, por lo tanto, urocultivo positivo. El crecimiento de dos o más microorganismos se consideró muestra contaminada, con base en las normas del Clinical and Laboratory Standards Institute (CLSI).,11-14

Se tomó como parámetro de multirresistencia bacteriana (MRB) a las bacterias resistentes a por lo menos 1 antibiótico en 3 o más categorías ${ }^{5}$ y resistencia panfarmacológica a bacterias resistentes a todos los antibióticos del antibiograma ordinario. $^{7}$

La multirresistencia bacteriana en Pseudomonas aeruginosa se definió como la resistencia a carbapenémicos, fluoroquinolonas y aminoglucosidos. ${ }^{15}$

El grupo de antimicrobianos del antibiograma analizado fue: amikacina, ampicilina-sulbactam, aztreonam, cefalotina, cefepime, cefotaxima, ceftazidima, ceftriaxona, ciprofloxacina, gentamicina, imipenem, levofloxacina, meropenem, piperacilina-tazobactam, tetraciclina, tobramicina y trimetoprima-sulfametoxazol.

Se filtraron los urocultivos con un solo organismo (por el sesgo conocido de contaminación al tener crecimiento de múltiples bacterias) hasta obtener solo los que mostraron resistencia a todo el grupo de antibióticos y con los registros se procedió a evaluar los expedientes físicos y electrónicos; se completaron las hojas de recolección de información, que incluyeron las variables consideradas para este estudio: sexo, edad, procedencia, lugar de tratamiento, enfermedades relacionadas, antecedente de cirugías, servicio a cargo, motivo de la hospitalización, trasfusión, tratamiento, portador de sonda urinaria, cultivo de control y defunción.

Para el análisis de los datos se utilizó estadística descriptiva. Con los datos obtenidos se elaboraron gráficas para evidenciar los resultados.

\section{RESULTADOS}

De acuerdo con la base de datos médicos, se analizaron 8164 pacientes con cultivos de orina positivos entre 2007 y 2015. En ese periodo la metodología y técnicas para identificar las bacterias fueron las mismas. El filtro por patrón de resistencia bacteriana identificó 44 microorganismos con resistencia a todo el grupo de antibióticos. La base de datos se redujo a 31 pacientes por datos incompletos en el expediente y por no encontrar expedientes en el archivo clínico; por tanto, se descartaron del estudio 13 pacientes.

Las infecciones provocadas por superbacterias afectaron con mayor frecuencia a los hombres. El promedio de edad fue de 61.9 (límites: 21 a 90 años), por lo que se ubicaron en el grupo de adultos mayores. La mayoría de los pacientes procedía del medio urbano y recibieron tratamiento en el hospital. Cuadro 1

Entre las enfermedades relacionadas, la diabetes, hipertensión y secuelas de enfermedades neurológicas fueron las más frecuentes en este tipo de infección. Figura 1

Los procedimientos quirúrgicos inmediatos a la infección se relacionaron con cirugía general y neurocirugía. La mayor parte de los servicios responsables de este tipo de pacientes fueron: medicina interna $(64.5 \%)$ y terapia intensiva $(12.9 \%)$. Las principales razones de hospitalización fueron: sepsis (38.7\%), infección de la vía urinaria $(29 \%)$ y neumonía $(9.6 \%)$. Como dato interesante, $58.06 \%$ de los pacientes tenía antecedente de trasfusión reciente al fenómeno estudiado.

Aunque no se contaba con algún antibiótico efectivo, se indicó tratamiento a todos los pacientes, ya sea por otro foco infeccioso o por 
Chavolla-Canal AJ y col. Infección de la vía urinaria por superbacterias

Cuadro 1. Variables de estudio (continúa en la siguiente página)

\section{Variable}

Subtipo

n

$\%$ (promedio)

Población con superbacterias en

Subtipo

la via urinaria

\begin{tabular}{|c|c|c|c|}
\hline \multirow{2}{*}{ Sexo } & Masculino & 21 & $67.74 \%$ \\
\hline & Femenino & 10 & $32.25 \%$ \\
\hline Edad & Promedio (límites) & & $61.93(21-90)$ \\
\hline \multirow{2}{*}{ Procedencia } & Rural & 7 & $22.58 \%$ \\
\hline & Urbano & 24 & $77.41 \%$ \\
\hline \multirow{3}{*}{ Lugar de tratamiento } & Hospital & 21 & $67.74 \%$ \\
\hline & Ambulatorio & 7 & $22.58 \%$ \\
\hline & Domicilio & 3 & $9.67 \%$ \\
\hline \multirow{7}{*}{ Enfermedades relacionadas } & Diabetes & 18 & $58.06 \%$ \\
\hline & Hipertensión & 13 & $41.93 \%$ \\
\hline & Secuelas neurológicas & 10 & $32.25 \%$ \\
\hline & Enfermedad prostática & 5 & $16.12 \%$ \\
\hline & Insuficiencia renal & 5 & $16.12 \%$ \\
\hline & $\begin{array}{l}\text { Enfermedad broncopulmonar } \\
\text { obstructiva crónica }\end{array}$ & 2 & $6.45 \%$ \\
\hline & Otras & 3 & $9.67 \%$ \\
\hline \multirow{5}{*}{ Cirugías } & Cirugía general & 4 & $12.9 \%$ \\
\hline & Neurocirugía & 4 & $12.9 \%$ \\
\hline & Urología & 2 & $6.4 \%$ \\
\hline & Traumatología y ortopedia & 2 & $6.4 \%$ \\
\hline & Ginecología & 1 & $3.2 \%$ \\
\hline \multirow{8}{*}{ Servicio a cargo } & Medicina interna & 20 & $64.51 \%$ \\
\hline & Terapia intensiva & 4 & $12.9 \%$ \\
\hline & Nefrología & 2 & $6.4 \%$ \\
\hline & Urología & 1 & $3.2 \%$ \\
\hline & Traumatología & 1 & $3.2 \%$ \\
\hline & Neurología & 1 & $3.2 \%$ \\
\hline & Infectología & 1 & $3.2 \%$ \\
\hline & Coloproctología & 1 & $3.2 \%$ \\
\hline \multirow{4}{*}{ Motivo de hospitalización } & Sepsis & 12 & $38.70 \%$ \\
\hline & Infección de la vía urinaria & 9 & $29.03 \%$ \\
\hline & Neumonía & 3 & $9.6 \%$ \\
\hline & Otras & 7 & $22.5 \%$ \\
\hline \multirow[t]{2}{*}{ Trasfusión } & & 18 & $58.06 \%$ \\
\hline & Cefalosporinas & 11 & $35.48 \%$ \\
\hline \multirow[t]{2}{*}{ Tratamiento } & Quinolonas & 9 & $29.03 \%$ \\
\hline & Carbapenémicos & 7 & $22.58 \%$ \\
\hline
\end{tabular}


Cuadro 1. Variables de estudio (continuación)

\begin{tabular}{|c|c|c|c|}
\hline Variable & Subtipo & $\mathbf{n}$ & $\%$ (promedio) \\
\hline & Vancomicina & 5 & $16.12 \%$ \\
\hline & Nitrofurantoína & 3 & $9.6 \%$ \\
\hline & Trimetoprima-sulfametoxazol & 1 & $3.2 \%$ \\
\hline & Linezolid & 1 & $3.2 \%$ \\
\hline & Amikacina & 1 & $3.2 \%$ \\
\hline \multirow{2}{*}{ Portador de sonda urinaria } & Sí & 23 & $74.19 \%$ \\
\hline & No & 8 & $25.80 \%$ \\
\hline \multirow{2}{*}{ Cultivo de control } & No & 25 & $80.64 \%$ \\
\hline & Sí & 6 & $19.35 \%$ \\
\hline Mortalidad & & 12 & $38.70 \%$ \\
\hline
\end{tabular}

un intento de erradicar la infección con cierto medicamento incluido en el panel de antibiograma básico del hospital. Con base en esto, los antibióticos más prescritos fueron: cefalosporinas (35.5\%), quinolonas (29\%), carbapenémicos $(22.6 \%)$ y vancomicina $(16.1 \%)$. Figura 2

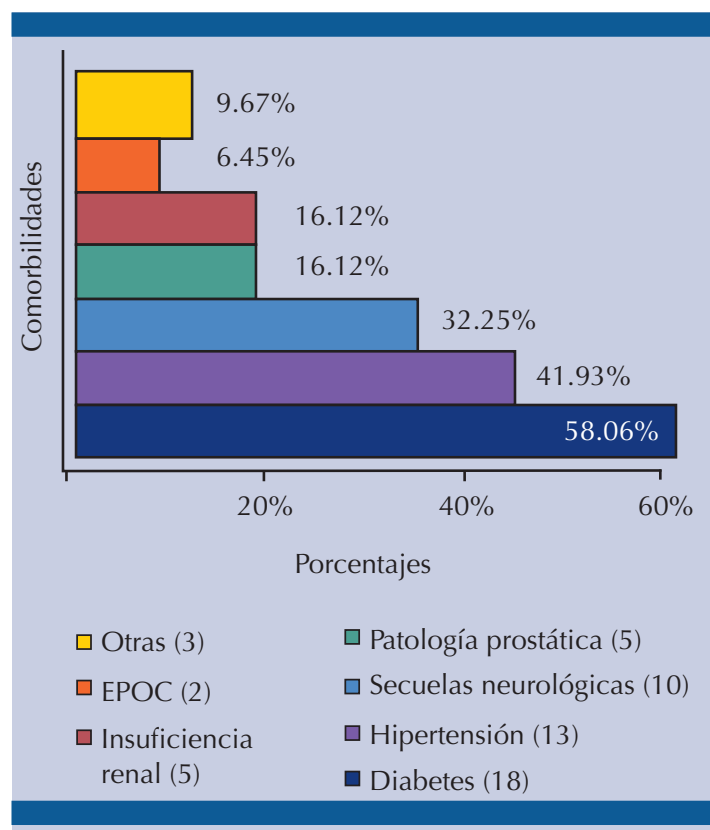

Figura 1. Enfermedades en pacientes con antecedente de infección de la vía urinaria provocada por superbacterias.
Pseudomonas aeruginosa fue la superbacteria responsable de la mayor parte de las infecciones de la vía urinaria. Figura 3

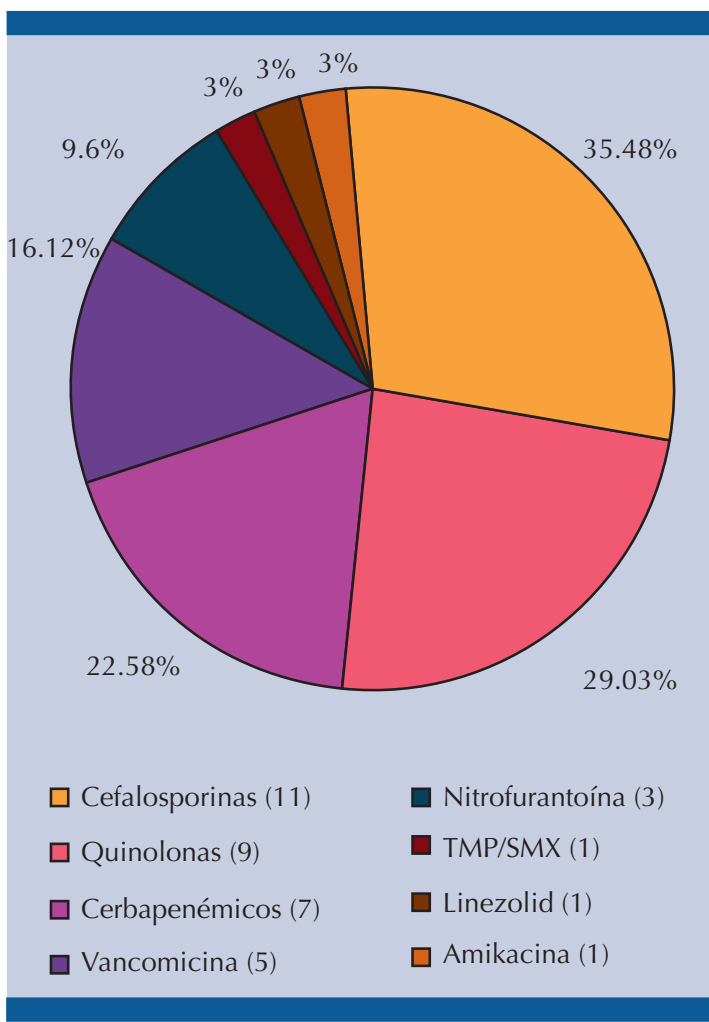

Figura 2. Prescripción de antibióticos a pacientes con infección por superbacterias.

TMP/SMX: Trimetoprima-sulfametoxazol 


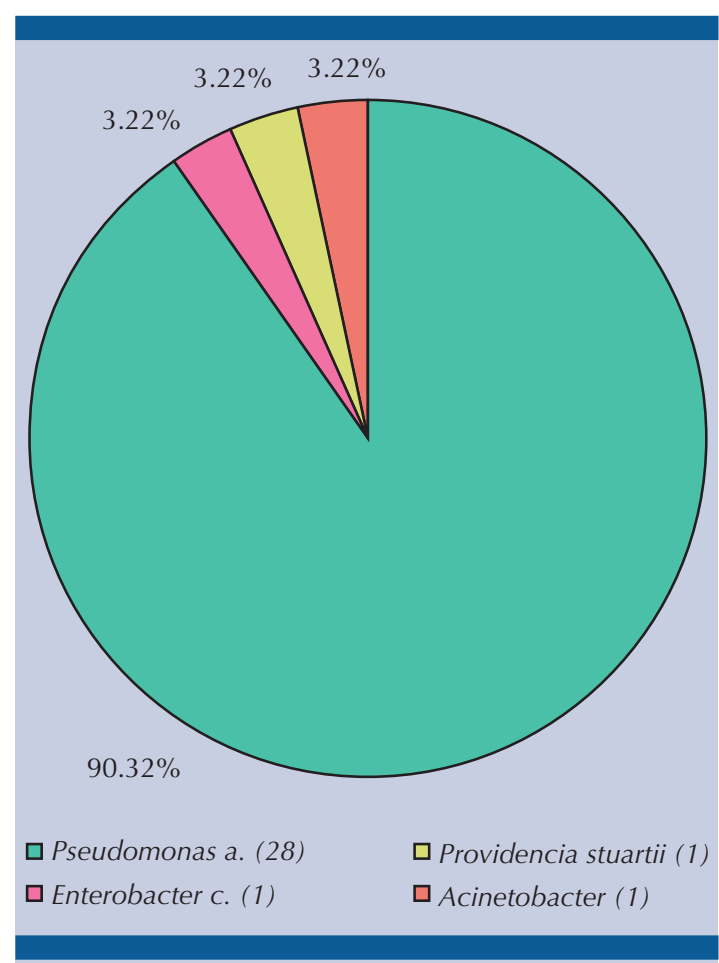

Figura 3. Bacterias aisladas de urocultivos con resistencia panfarmacológica.

De las variables analizadas, se registraron 23/31 pacientes portadores de sonda urinaria. Aunque las infecciones fueron tratadas de manera empírica, solo se realizó cultivo de control en 6 casos y de éstos 2 tuvieron erradicación de la superbacteria.

La mortalidad por infección en la población estudiada fue de 12/31 casos. Al analizar las variables en la subpoblación de los pacientes que fallecieron (10/12 hombres), la que tuvo mayor relación fue la trasfusión, seguida de la colocación de sonda urinaria, hospitalización en Medicina interna, sepsis a su ingreso, diabetes e hipertensión (Cuadro 2). En cuanto a la mortalidad por grupos, se registró un mayor índice en quienes tuvieron sepsis, seguido de trasfusión y pacientes atendidos en el área de Medicina interna (Figura 4).
Cuadro 2. Principales causas de mortalidad

\begin{tabular}{|l|c|c|}
\hline Variables & Mortalidad (n) & $\%$ \\
\hline Mortalidad general & 12 & $100 \%$ \\
\hline Trasfusión & 11 & $91.6 \%$ \\
\hline Sonda & 10 & $83.3 \%$ \\
\hline Sexo masculino & 10 & $83.3 \%$ \\
\hline Hospitalización en & 9 & $75 \%$ \\
Medicina interna & & \\
\hline Sepsis & 8 & $66.6 \%$ \\
\hline Diabetes & 8 & $66.6 \%$ \\
\hline Hipertensión & 5 & $41.6 \%$ \\
\hline
\end{tabular}

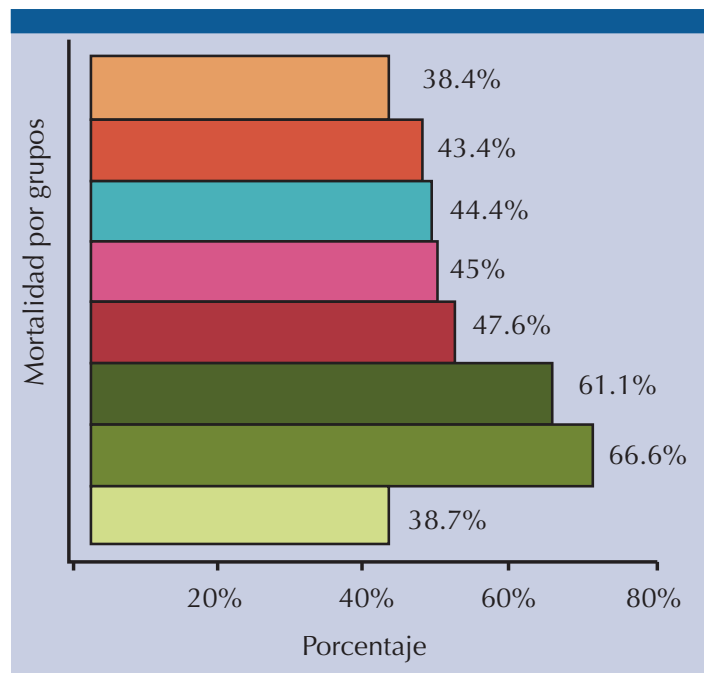

口 Hipertensión (5 de 13)

$\square$ Sonda (10 de 23)

- Sexo masculino (10 de 21)

$\square$ Diabetes (8 de 18)

- Trasfusión (11 de 18)

$\square$ Hospitalización en

- Sepsis (8 de 12)

Medicina interna (9 de 20)

$\square$ Mortalidad general (12 de 31)

Figura 4. Mortalidad relacionada con las variables de estudio por grupo.

No se encontró relación importante de variables para supervivencia, excepto en el cultivo de control con posterior curación de la infección (n =2), que haberlo realizado en 6/31 casos, esta variable pierde su relevancia. 


\section{DISCUSIÓN}

Este estudio reportó $0.53 \%$ de infecciones por superbacterias, lo que representa un porcentaje bajo, comparado con otras investigaciones a nivel mundial. Debido a la reducción del tamaño de la muestra, por la falta de información al tener expedientes incompletos, no fue posible realizar un análisis de calidad. De acuerdo con el análisis de los resultados, la infección panfarmacológica de vías urinarias es más frecuente en hombres y provoca mayor mortalidad que en las mujeres, incluso suele afectar a la población de la tercera edad, pero no es exclusiva de este grupo, ya que se registraron casos en pacientes relativamente jóvenes. El lugar de procedencia de la población en estudio fue mayormente urbano, este dato es importante porque supone que al pertenecer a un medio de fácil acceso a los servicios de salud, se exponen de manera habitual al consumo inconsciente o indiscriminado de antibióticos, que tiene repercusión importante en la resistencia bacteriana.

Las enfermedades más frecuentes identificadas en este estudio coinciden con las descritas en ensayos de multirresistencia: diabetes con subsiguiente afectación del sistema inmunológico, entre otros factores, para favorecer la resistencia bacteriana. El servicio de Medicina interna fue el sitio con mayor atención de pacientes y mortalidad más alta. Los pacientes que ingresaron con datos de sepsis fueron quienes registraron mayor índice de mortalidad, quizá debido a la mortalidad intrínseca de la respuesta sistémica, además del aislamiento de alguna bacteria con resistencia panfarmacológica. ${ }^{1,6,8-13}$

Ningún estudio reporta que la trasfusión se relaciona con mortalidad; sin embargo, en el estudio aquí realizado los pacientes de este grupo registraron la tasa más alta de fallecimientos. No se conoce la relación de la alta mortalidad en este grupo, ni la conexión con bacterias más resistentes, por lo que se requieren estudios adicionales para establecer una relación más clara y saber si va más allá de los riesgos inherentes a la propia trasfusión.

Por no contar con cultivos de control evidenciamos una falla común en la evolución de las infecciones urinarias en nuestra región. De acuerdo con la bibliografía de todo el mundo, el factor asociado para adquirir infecciones urinarias, asociadas con bacterias resistentes, es la colocación de sondas urinarias, donde Pseudomonas aeruginosa es el microorganismo aislado con más frecuencia (Cuadro 1 y Figura 3). ${ }^{10} \mathrm{La}$ mortalidad general por infección provocada por superbacterias se encontró en un porcentaje inferior al reportado en la bibliografía, pues algunas investigaciones registran tasas de mortalidad, incluso, de $80 \%$. $^{1,6,8-13}$

Los factores de riesgo para infecciones de la vía urinaria por bacterias de resistencia panfarmacológica y con alta mortalidad fueron: trasfusiones, colocación de sonda urinaria, pacientes diabéticos, sexo masculino, hospitalización en el área de medicina interna, sepsis e hipertensión. Por tanto, debe tenerse especial cuidado en los pacientes con alguno de estos factores, además de urocultivo con especificación de resistencia bacteriana en todo el grupo de antibióticos.

\section{CONCLUSIONES}

Las infecciones de la vía urinaria provocadas por superbacterias tienen baja prevalencia, menor mortalidad respecto de otras poblaciones y afectan, principalmente, a hombres, a pacientes con alteraciones del sistema inmunitario, con sonda urinaria y con trasfusión de hemoderivados. Los datos obtenidos justifican un tratamiento más agresivo y urgente cuando se identifique alguna de las variables relacionadas con alta mortalidad en un paciente con características de infección por superbacterias. También se sugiere mayor 
Chavolla-Canal AJ y col. Infección de la vía urinaria por superbacterias

cuidado al colocar sondas urinarias, esmerarse en el aseo de manos e indicar todas las medidas de seguridad para el paciente. Una limitante de esta investigación es el número reducido de pacientes, por lo que se requieren estudios prospectivos, que consideren variables adicionales, incluso, estudio genético de las superbacterias para el seguimiento del fenómeno de adaptación a la resistencia bacteriana.

\section{REFERENCIAS}

1. Solorzano A, et al. Evolution of the resistance to antibiotic of bacteria involved in urinary tract infections: A 7-year surveillance study. Am J Infect Control 2014;42:1033-8. DOI: 10.1016/j.ajic.2014.06.013

2. Ankur-Tyagi $\mathrm{V}$, et al. Isolation and antibacterial susceptibility testing of multi drug resistant Pseudomonas aeruginosa causing urinary tract infections. J Chem Pharm Res 2011;3(4):342-347.

3. Informe Epidemiológico de la Secretaria de Salud, México 2014. Incidencia de infección de vías urinarias por grupos de edad. Estados Unidos Mexicanos 2014. Población General.

4. Rodriguez-Noriega E, et al. La evolución de la resistencia bacteriana en México, 1973-2013. Biomédica 2014;34(Supl.1):181-90. DOI: http://dx.doi.org/10.7705/ biomedica.v34i0.2142.

5. Shah DA, et al. Antibiotic resistance pattern of Pseudomonas aeruginosa isolated from urine samples of urinary tract infections patients in Karachi, Pakistan. Pak J Med Sci. 2015; 2:341-345. DOI: 10.12669/pjms.312.6839

6. De Francesco MA, et al. Prevalence of multidrug-resistant Acinetobacter baumannii and Pseudomonas aeruginosa in an Italian hospital. J Infect Public Health 2013;6(3):179-185. DOI: 10.1016/j.jiph.2012.11.006

7. Pereira SG, et al. Multidrug and extensive drug resistance in Pseudomonas aeruginosa clinical isolates from a Portuguese central hospital: 10-year survey. Microb drug Resist 2015;2:194-200. DOI: 10.1089/mdr.2014.0137

8. OSSA-GIRALDO, Ana Claudia et al. Factores de riesgo para infección por Pseudomonas aeruginosa multi-resistente en un hospital de alta complejidad. Rev Chil Infectol 2014;31(4):393-399. http://dx.doi.org/10.4067/S071610182014000400003.

9. Cornejo-Juárez $\mathrm{P}$, et al. Patrones de resistencia bacteriana en urocultivos en un hospital oncológico. Salud Pub Mex 2007;49(5):330-336.

10. Bjerklund-Johansen TE, et al. Prevalence of hospital-acquired urinary tract infections in urology departments. Eur Urol 2007;51(4):1100-11. DOI: 10.1016/j.eururo.2006.08.012

11. Grabe M, et al. Guidelines on Urological Infections. European Association of Urology 2015;9-35 [en línea]. Dirección URL: <https://uroweb.org/wp-content/uploads/19-Urologicalinfections_LR2.pdf $>$.

12. McDougal W, et al. Campbell-Walsh Urology $13^{\text {th }}$ edition. In: Ewein AJ, et al. Philadelphia: Saunders-Elsevier, 2012;257.

13. Peña $C$, et al. Risk-factors for acquisition of extendedspectrum b-lactamase-producing Escherichia coli among hospitalised patients. Clin Microbiol Infect 2006;12:279-284. http://dx.doi.org/10.1111/j.1469-0691.2005.01358.x

14. Clinical and Laboratory Standards Institute. Performance standards for antimicrobial susceptibility testing; twentysecond information supplement. Wayne(PA): Clinical and Laboratory Standards Institute ; 2012. Publication M-100-S22.

15. Chavolla A, et al. Prevalencia de bacterias aisladas con resistencia antibiótica extendida en los cultivos de orina durante 8 años en un hospital de segundo nivel en México. Rev Mex Urol 2016;76(4):213-217. DOI: http://dx.doi: 10.1016/j. uromx.2016.04.00 